\title{
Acute flaccid myelitis associated with enterovirus-D68 infection in an otherwise healthy child
}

Susanna Esposito ${ }^{1 *}$, Giovanna Chidini ${ }^{2}$, Claudia Cinnante ${ }^{3}$, Luisa Napolitano ${ }^{2}$, Alberto Giannini ${ }^{2}$ Leonardo Terranovaa ${ }^{1}$, Hubert Niesters ${ }^{4}$, Nicola Principi ${ }^{1}$ and Edoardo Calderini ${ }^{2}$

\begin{abstract}
Background: Reporting new cases of enterovirus (EV)-D68-associated acute flaccid myelitis (AFM) is essential to understand how the virus causes neurological damage and to characterize EV-D68 strains associated with AFM.

Case presentation: A previously healthy 4-year-old boy presented with sudden weakness and limited mobility in his left arm. Two days earlier, he had an upper respiratory illness with mild fever. At admission, his physical examination showed that the child was febrile $\left(38.5^{\circ} \mathrm{C}\right)$ and alert but had a stiff neck and weakness in his left arm, which was hypotonic and areflexic. Cerebrospinal fluid (CSF) examination showed a mild increase in white blood cell count $\left(80 / \mathrm{mm}^{3}, 41 \%\right.$ neutrophils) and a slightly elevated protein concentration $(76 \mathrm{gm} / \mathrm{dL})$. Bacterial culture and molecular biology tests for detecting viral infection in CSF were negative. The patient was then treated with intravenous ceftriaxone and acyclovir. Despite therapy, within $24 \mathrm{~h}$, the muscle weakness extended to all four limbs, which exhibited greatly reduced mobility. Due to his worsening clinical prognosis, the child was transferred to our Pediatric Intensive Care Unit; at admission he was diagnosed with acute flaccid paralysis of all four limbs. Brain magnetic resonance imaging (MRI) was negative, except for a focal signal alteration in the dorsal portion of the medulla oblongata, also involving the pontine tegmentum, whereas spine MRI showed an extensive signal alteration of the cervical and dorsal spinal cord reported as myelitis. Signal alteration was mainly localized in the central grey matter, most likely in the anterior horns. Molecular biology tests performed on nasopharyngeal aspirate and on bronchoalveolar lavage fluid were negative for bacteria but positive for EV-D68 clade B3. Plasmapheresis was performed and corticosteroids and intravenous immunoglobulins were administered. After 4 weeks of treatment, the signs and symptoms of AFM were significantly reduced, although some weakness and tingling remained in the patient's four limbs. MRI acquired after 3 weeks showed that the previously reported alterations were no longer present.
\end{abstract}

Conclusion: This case suggests that EV-D68 is a neurotropic agent that can cause AFM and strains are circulating in Europe. EV-D68 disease surveillance is required to better understand EV-D68 pathology and to compare various strains that cause AFM.

Keywords: Acute flaccid paralysis, Enterovirus D68, Neurological infection

\footnotetext{
* Correspondence: susanna.esposito@unimi.it

${ }^{1}$ Pediatric Highly Intensive Care Unit, Department of Pathophysiology and

Transplantation, Università degli Studi di Milano, Fondazione IRCCS Ca'

Granda Ospedale Maggiore Policlinico, Via Commenda 9, 20122 Milano, Italy

Full list of author information is available at the end of the article
} 


\section{Background}

Until 2013, enterovirus (EV)-D68 was considered a rare cause of disease because only sporadic cases and minor outbreaks of respiratory infection occurred. However, since 2014, EV-D68 infection has gained epidemiological and clinical relevance when the virus caused a large-scale outbreak of severe respiratory infections, mainly in children, in the USA and Canada, with subsequent cases in other countries [1-3]. Respiratory disorders due to EVD68 varied from pharyngitis and bronchitis to severe pneumonia with respiratory failure [4]. Additionally, concomitant with EV-D68-associated respiratory disease in the USA, there was an increase in the number of cases of severe neurological disease, mainly acute flaccid myelitis (AFM) [5]. EV-D68 was not detected in the cerebrospinal fluid (CSF) of these patients, and therefore definitive causation between EV-D68 and AFM was not established. However, the possibility of EV-D68 causing a polio-like disease was still considered. Other EVs, specifically EVA71, are well known neurotropic agents that can cause aseptic meningitis, meningoencephalitis, and AFM [6]. Moreover, EV-D68 was detected in the respiratory secretions of several of the AFM cases diagnosed during the 2014 outbreak in the USA [5]. Finally, a case-control study of the presence of EV-D68 in upper respiratory secretions of children with acute respiratory infection demonstrated that children with infection and AFM were 10.3 times more likely to be infected with EV-D68 than children without AFM tested for respiratory infections [7]. Very few EV-D68-associated AFM cases have been reported in Europe [8-12]. Reporting new cases of this disease is essential to better understand how EV-D68 causes neurological damage and to identify and characterize the strains associated with AFM. In this paper, a case of AFM associated with EV-D68 infection occurring in an otherwise healthy child in Milan, Italy, is described.

\section{Case presentation}

A previously healthy, fully immunized, 4-year-old boy came to the Emergency Room of the Saint Anna Hospital in Como on July 21, 2016, presenting with sudden onset weakness and poor mobility in his left arm. Two days earlier, he had an upper respiratory illness with mild fever, rhinorrhoea, and cough. Past and recent clinical histories were negative for relevant diseases. At admission, physical examination of the patient showed that the child had a temperature of $38.5^{\circ} \mathrm{C}$, a heart rate of $131 / \mathrm{min}$, a respiratory rate of $18 / \mathrm{min}, 98 \%$ oxygen saturation and a blood pressure of 110/70 $\mathrm{mmHg}$. Respiratory, cardiovascular, and skin examinations were normal. Neurological evaluation demonstrated that the child was alert but had a stiff neck and weakness in his left arm, which was hypotonic and areflexic. The results of cranial nerve examination, including fundoscopy, and diagnoses of the right arm and bilateral leg strength were normal.
Results from a complete blood count were in the normal range, with the exception of slightly elevated white blood cell and neutrophil counts. Serum electrolytes, coagulation factors, liver enzymes, and C-reactive protein were within the normal limits. Fine bilateral perihilar infiltration was observed on a chest X-ray to give the diagnosis of probable community-acquired pneumonia of viral aetiology.

CSF examination showed a mild increase in white blood cell count $\left(80 / \mathrm{mm}^{3}, 41 \%\right.$ neutrophils) with normal glucose (60 mg/dL) and slightly elevated protein (76 gm/dL) concentrations. Cultures for bacteria were negative, and molecular biology tests for detecting viral infection were negative for herpes simplex virus 1 , herpes simplex virus 2 , human herpesvirus 6, EV, varicella zoster virus, adenovirus, parvovirus, cytomegalovirus, and Epstein-Barr virus. Computed tomography of the head without contrast did not show abnormalities. Intravenous ceftriaxone and acyclovir were then administered.

Despite this therapy, the child's neurological function deteriorated rapidly. Within $24 \mathrm{~h}$, the muscle weakness extended to all four limbs, which exhibited greatly reduced mobility. Due to his worsening clinical prognosis, the child was transferred to the Pediatric Intensive Care Unit (PICU) of the Fondazione IRCCS Ca' Granda, Ospedale Maggiore Policlinico, Milan, Italy.

When the child was admitted to the PICU on July 22, 2016, he was alert but was diagnosed with acute flaccid paralysis based on neurological examination. Together with the muscles of the four limbs, the respiratory muscles were also clearly involved. Moreover, a deficit of the right facial nerve with dysphagia was observed. The child required intubation and mechanical ventilation for adequate gas exchange. After 11 days, tracheotomy was performed. Nutrition was assured through percutaneous endoscopic gastrotomy.

Figure $1 \mathrm{a}-\mathrm{d}$ summarizes the results of magnetic resonance imaging (MRI) of the brain at admission to the PICU, 3 days and about $24 \mathrm{~h}$ after the onset of respiratory and neurological manifestations, respectively. A focal signal alteration was found localized in the dorsal portion of the medulla oblongata, also involving the pontine tegmentum, without enhancement or diffusion restriction. Moreover, MRI of the spinal cord showed an extensive signal alteration of the cervical and dorsal spinal cord, reported as myelitis. The signal alteration was mainly localized in the central grey matter, most likely in the anterior horns. After contrast administration, a slight enhancement in some of the caudal roots was observed, without enhancement in the spinal cord.

The presence of somatosensory-evoked potentials confirmed that only the motor pathway was affected. Acute polyradiculoneuritis was excluded because there was no albumino-cytological dissociation and no antiganglioside antibodies in the CSF. There was no evidence of paraneoplasic syndrome or inflammatory disease. 


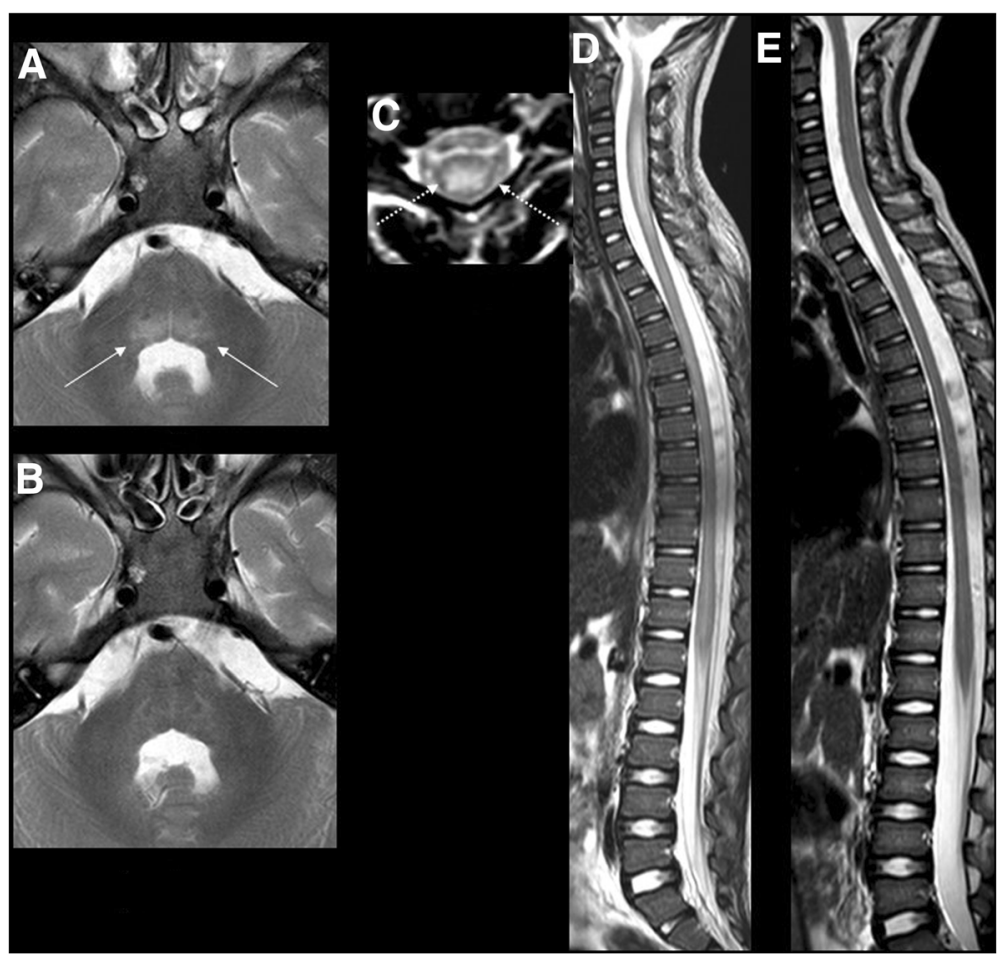

Fig. 1 Magnetic resonance imaging (MRI) of the brain and spinal cord: a, axial T2 image at the level of the pons showing an increased signal in the tegmentum (white arrows), which was completely resolved in the follow up scan acquired 3 weeks later; $\mathbf{b}$, sagittal and axial T2 image of the spinal cord (c, d) showing cord swelling, particularly at the cervical level (white, dot arrow), with extensive hyperintensity in the central cord (d), which was also completely resolved in the follow up images (e) acquired 3 weeks later

Bacterial cultures of the nasopharyngeal aspirate and the bronchoalveolar lavage fluid were negative. Molecular biology tests for detecting viral infection were negative for herpes simplex virus 1 , herpes simplex virus 2 , human herpesvirus 6, varicella zoster virus, adenovirus, parvovirus, cytomegalovirus and EBV, but was positive for EV. Both the respiratory samples were reanalysed for the presence of EVD68 using single-tube real-time PCR to amplify a $116 \mathrm{bp}$ fragment of the $5^{\prime}$ non-translated region according to the method proposed by Poelman et al. [13]. Phylogenetic analysis based on the method used by Nix et al. [14] verified the presence of a virus belonging to clade B3 of the EVD68 phylogenetic tree (Fig. 2). Sequence analysis showed that the 2016 strains were closely related to sequences of the recently described subclade B3, represented in Fig. 2, by four sequences obtained in China. The level of nucleotide divergence was $2.1 \%$ within $\mathrm{B} 3,5.5 \%$ between $\mathrm{B} 1$ and $\mathrm{B} 3$, and 7.3\% between B2 and B3. The sequence from our Italian AFM case was closely related to Dutch AFM EVD68 cases from 2016.

Serological analysis showed no infection with herpes simplex virus, varicella zoster virus, Epstein-Barr virus, cytomegalovirus, Mycoplasma pneumoniae, Borrelia burgdorferi, Cryptococcus neoformans, or Mycobacterium tuberculosis.
Treatment with intravenous methylprednisolone $(30 \mathrm{mg}$ / $\mathrm{kg}$ ) was initiated. Plasmapheresis was conducted and intravenous immunoglobulins ( $1 \mathrm{~g} / \mathrm{kg} /$ day $)$ were administered during the first 3 days in the PICU. Intravenous steroid therapy was suspended after 5 days and substituted with oral prednisone ( $2 \mathrm{mg} / \mathrm{kg} /$ day) for 4 weeks, which was then tapered over an additional 2 weeks.

Significant weakness with reduced mobility of the four limbs and difficulty swallowing persisted with very slow regression. After 4 weeks of treatment, all the signs and symptoms of AFM were significantly reduced or disappeared, although a certain degree of weakness and tingling in the four extremities were still present. Moreover, deep tendon reflexes were generally reduced. However, as expected due to the recent onset of the disease, no muscle atrophy was observed. Moreover, the results of MRI performed about 1 month after the onset of the first neurological manifestations showed that the previously reported alterations were no longer present (Fig. 1e).

\section{Conclusions}

AFM is a rare disease in polio-free geographical areas. Most of the cases are due to EVs, mainly EV-A71, flaviviruses, Japanese encephalitis virus, and West Nile virus $[15,16]$. Recently, several AFM cases were diagnosed during an 




Fig. 2 Phylogenetic tree: Sequence analysis showed that the strains obtained in 2016 (in red) are closely related to sequences of the recently described subclade $B 3$, represented by four sequences from China (in green, 2014). The sequence from our Italian acute flaccid myelitis case (in light blue) is closely related to Dutch acute flaccid myelitis EV-D68 cases from 2016. Other 2014 cases are in dark blue. The nucleotide divergence was $2.1 \%$ within B3, 5.5\% between B1 and B3, and $7.3 \%$ between B2 and B3. As outlier, the Fermon strain from 1962 was used. Analysis was performed using Bionumerics Software (Biomerieux, France) with sequencing partial VP1

outbreak of EV-D68 respiratory infection, indicating an association between AFM and EV-D68 infection, although a direct causative role has not been established $[3,5]$. However, EV-D68 has been detected in the cerebrospinal fluid of two patients with AFM $[3,5,15,17]$ and, more recently, in two other patients with aseptic meningitis [18].

The case described here suggests that EV-D68 is a neurotropic agent that can cause AFP. The case of AFM described here clinically resembles those described in the USA and Canada since 2014 [7] and the few cases described later in Europe [16]. AFM was diagnosed in a child suffering from a mild acute respiratory infection, who was febrile at the onset of neurological symptoms. Moreover, the pattern of neurological deficits and neuroimaging abnormalities localizing to the anterior horn cells of the spinal cord and cranial nerve motor nuclei in the brainstem are similar to those observed in other patients with AFM. Finally, CSF examination revealed alterations suggestive of aseptic meningitis. Together with these findings, the presence of EV-D68 in both the nasopharyngeal aspirate and BAL of the patient suggests a relationship between AFM and EV-D68, particularly because no other infection of the central nervous system could be found. The inability to detect EV-D68 in the CSF does not greatly weaken this relationship because an inability to detect an infectious agent in the central nervous system of patients with neurological complications from neurotropic viruses, including polio and EV-A71, is common [6]. A progressive reduction in signs and symptoms of disease occurred, albeit slowly, in the child described here, and MRI performed approximately 1 month after the onset of disease did not show persistent alterations. Although electromyogram and nerve conduction studies better correlate than MRI with final prognosis of AFM, this result suggests that this case could have a favourable prognosis, with longterm resolution of the neurological problems.

However, some reported cases have a less favourable outcome. Two children with EV-D68-associated AFM in Norway were recently described as having persistent deficits [8]. One had impaired head and motor control in his arm and the second developed atrophy in the left upper arm and shoulder. Thus, neurological damage due to EVD68 infection can be severe and persistent, in a similar manner to polio infection and infection with other known neurotropic viruses.

The EV-D68 strain detected in this study was found to belong to the B3 clade. Despite all three clades of EV-D68 having been found to circulate worldwide, most of the cases detected in the USA [19] and Europe [3, 20] were from genetically similar viruses of clade B. Data regarding strains associated with the development of AFM are scarce. However, strains identified in the USA [16] were found to differ from other clade B strains in six amino acid substitutions, three of which are found in the structural and nonstructural proteins, thereby further defining the B1 clade. This finding led to the hypothesis that these strains could be significantly more neurotropic and virulent than other EV-D68 strains and that sequence data of the infecting strain could predict the clinical manifestations and outcomes of EV-D68 infection [21]. Our case report did not support this hypothesis and indicates that sequence data cannot predict clinical outcome. However, although characterization of EV-D68 is usually based on sequencing the VP1 region, mutations in other regions might confer higher virulence and increased risk of neurological complications during infection with certain EVD68 strains. EV-D68 disease surveillance is warranted to clarify any potential differences in strain virulence and to define the pathogenic role of infection as a cause of AFM.

\section{Abbreviations \\ AFM: Acute flaccid myelitis; CSF: Cerebrospinal fluid; EV: Enterovirus; MRI: Magnetic resonance imaging; PICU: Pediatric intensive care unit}

\section{Acknowledgements}

We thank Fabio Triulzi, Giovanna Lunghi, Samantha Bosis, Claudia Tagliabue, and Randy Poelman for their support in patient management. The authors thank the patient and his parents for allowing us to publish this case report. 


\section{Funding}

This case report was supported by a grant from the Italian Ministry of Health (Ricerca Corrente Grant 2016 850/01).

\section{Availability of data and materials}

All the data and materials used in this report are included in the manuscript.

\section{Authors' contributions}

SE provided infectious disease consultation to the child, supervised laboratory analyses, and co-drafted the manuscript; GC, LN, AG and EC followed-up the patient during hospitalization; CC performed MRI; LT performed laboratory assays; HN supervised phylogenetic analyses; NP coordinated laboratory analyses, co-drafted the manuscript and made substantial scientific contributions. All authors have read and approved the final version of the manuscript.

\section{Competing interests}

The authors declare that they have no competing interests.

\section{Consent for publication}

Written informed consent for the publication of this case report and any accompanying images was obtained from the patient's parents. A copy of the written consent is available for review by the Editor-in-Chief of this journal.

\section{Ethics approval and consent to participate}

This case report was approved by the Ethics Committee of Fondazione IRCCS Ca' Granda Ospedale Maggiore Policlinico, Milan, Italy. For case reports, the Ethics Committee of Fondazione IRCCS Ca' Granda Ospedale Maggiore Policlinico does not provide a reference number.

\section{Author details}

${ }^{1}$ Pediatric Highly Intensive Care Unit, Department of Pathophysiology and Transplantation, Università degli Studi di Milano, Fondazione IRCCS Ca' Granda Ospedale Maggiore Policlinico, Via Commenda 9, 20122 Milano, Italy. 2Pediatric Intensive Care Unit, Fondazione IRCCS Ca' Granda Ospedale Maggiore Policlinico, Milan, Italy. ${ }^{3}$ Neuroadiology Unit, Department of Pathophysiology and Transplantation, Università degli Studi di Milano, Fondazione IRCCS Ca' Granda Ospedale Maggiore Policlinico, Milan, Italy. ${ }^{4}$ Department of Medical Microbiology, Division of Clinical Virology, University Medical Center Groningen, Groningen, The Netherlands.

\section{Received: 27 September 2016 Accepted: 28 December 2016}

\section{Published online: 11 January 2017}

\section{References}

1. Holm-Hansen CC, Midgley SE, Fischer TK. Global emergence of enterovirus D68: a systematic review. Lancet Infect Dis. 2016;16:e64-75.

2. Esposito S, Bosis S, Niesters H, Principi N. Enterovirus D68 infection. Viruses. 2015;7:6043-50.

3. Poelman R, Schuffenecker I, Van Leer-Buter C, Josset L, Niesters HG, Lina B, ESCVECDC EV-D68 study group. European surveillance for enterovirus D68 during the emerging North-American outbreak in 2014. J Clin Virol. 2015;71:1-9.

4. Principi N, Esposito S. Enterovirus D-68: an emerging cause of infection. Expert Rev Respir Med. 2015;9:711-9.

5. Ayscue $P$, Van Haren $K$, Sheriff $H$, Waubant E, Waldron P, Yagi S, Yen C, Clayton A, Padilla T, Pan C, Reichel J, Harriman K, Watt J, Sejvar J, Nix WA, Feikin D, Glaser C, Centers for Disease Control and Prevention (CDC). Acute flaccid paralysis with anterior myelitis - California, June 2012-June 2014. MMWR Morb Mortal Wkly Rep. 2014;63:903-6.

6. Huang $\mathrm{HI}$, Shih SR. Neurotropic Enterovirus infections in the central nervous system. Viruses. 2015;7:6051-66.

7. Aliabadi N, Messacar K, Pastula DM, Robinson CC, Leshem E, Sejvar JJ, Nix WA, Oberste MS, Feikin DR, Dominguez SR. Enterovirus D68 infection in children with acute flaccid myelitis, Colorado, USA, 2014. Emerg Infect Dis. 2016;22:1387-94.

8. Pfeiffer HC, Bragstad K, Skram MK, Dahl H, Knudsen PK, Chawla MS, HolbergPetersen M, Vainio K, Dudman SG, Kran AM, Rojahn AE. Two cases of acute severe flaccid myelitis associated with enterovirus D68 infection in children, Norway, autumn 2014. Euro Surveill. 2015;20:21062.

9. Williams CJ, Thomas RH, Pickersgill TP, Lyons M, Lowe G, Stiff RE, Moore C, Jones R, Howe R, Brunt H, Ashman A, Mason BW. Cluster of atypical adult Guillain-Barré syndrome temporally associated with neurological illness due to
EV-D68 in children, South Wales, United Kingdom, October 2015 to January 2016. Euro Surveill 2016;21. doi: 10.2807/1560-7917.ES.2016.21.4.30119.

10. Lang M, Mirand A, Savy N, Henquell C, Maridet S, Perignon R, Labbe A, Peigue-Lafeuille $\mathrm{H}$. Acute flaccid paralysis following enterovirus D68 associated pneumonia, France, 2014. Euro Surveill. 2014;19.

11. Antona D, Kossorotoff M, Schuffenecker I, Mirand A, Leruez-Ville M, Bassi C, Aubart M, Moulin F, Lévy-Bruhl D, Henquell C, Lina B, Desguerre I. Severe paediatric conditions linked with EV-A71 and EV-D68, France, May to October 2016. Euro Surveill. 2016;21.

12. Dyrdak R, Grabbe M, Hammas B, Ekwall J, Hansson KE, Luthander J, Naucler $P$, Reinius $H$, Rotzén-Östlund M, Albert J. Outbreak of enterovirus D68 of the new B3 lineage in Stockholm, Sweden, August to September 2016. Euro Surveill. 2016;21.

13. Poelman $R$, Schölvinck $E H$, Borger $H$, Niesters $H G$, van Leer-Buter $C$. The emergence of enterovirus D68 in a Dutch University Medical Center and the necessity for routinely screening for respiratory viruses. J Clin Virol. 2015;62:1-5.

14. Nix WA, Oberste MS, Pallansch MA. Sensitive, seminested PCR amplification of VP1 sequences for direct identification of all enterovirus serotypes from original clinical specimens. J Clin Microbiol. 2006;44:2698-704.

15. Khetsuriani N, Lamonte-Fowlkes A, Oberst S, Pallansch MA, Centers for Disease Control and Prevention. Enterovirus surveillance-United States, 1970-2005. MMWR Surveill Summ. 2006:55:1-20.

16. European Centre for Disease Prevention and Control. Rapid Risk Assessment - Enterovirus detections associated with severe neurological symptoms in children and adults in European countries, 8 August 2016. Stockholm: ECDC; 2016. Available at: http://ecdc.europa.eu/en/publications/Publications/01-082016-RRA-Enterovirus\%2071-Spain,\%20France,\%20Netherlands.pdf. Accessed 16 Sept 2016.

17. Kreuter JD, Barnes A, McCarthy JE, Schwartzman JD, Oberste MS, Rhodes $\mathrm{CH}$, Modlin JF, Wright PF. A fatal central nervous system enterovirus 68 infection. Arch Pathol Lab Med. 2011;135:793-6.

18. Esposito S, Lunghi G, Zampiero A, Tagliabue C, Orlandi A, Torresani E, Niesters $\mathrm{H}$, Principi N. Enterovirus-D68 in the cerebrospinal fluid of two children with aseptic meningitis. Pediatr Infect Dis J. 2016;35:589-91.

19. Greninger AL, Naccache SN, Messacar K, Clayton A, Yu G, Somasekar S, Federman S, Stryke D, Anderson C, Yagi S, Messenger S, Wadford D, Xia D, Watt JP, Van Haren K, Dominguez SR, Glaser C, Aldrovandi G, Chiu CY. A novel outbreak enterovirus D68 strain associated with acute flaccid myelitis cases in the USA (2012-14): a retrospective cohort study. Lancet Infect Dis. 2015;15:671-82

20. Van Leer-Buter CC, Poelman R, Borger R, Niesters HG. Newly identified Enterovirus $C$ genotypes, identified in the Netherlands through routine sequencing of all Enteroviruses detected in clinical materials from 2008 to 2015. J Clin Microbiol. 2016:54:2306-14.

21. Norder H, Magnius L. Can sequence data predict enterovirus D68 infection outcome? Lancet Infect Dis. 2015:15:620-1.

\section{Submit your next manuscript to BioMed Central and we will help you at every step:}

- We accept pre-submission inquiries

- Our selector tool helps you to find the most relevant journal

- We provide round the clock customer support

- Convenient online submission

- Thorough peer review

- Inclusion in PubMed and all major indexing services

- Maximum visibility for your research

Submit your manuscript at www.biomedcentral.com/submit
BioMed Central 